\title{
Magnetic flux gradient observation during fatigue crack propagation: A case study of SAE 1045 carbon steel used for automotive transmission parts
}

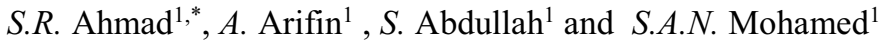 \\ ${ }^{1}$ Department of Mechanical and Materials Engineering, Faculty of Engineering and Built \\ Environment, Universiti Kebangsaan Malaysia, 43600 UKM Bangi,Selangor, Malaysia
}

\begin{abstract}
The objective of this study is to evaluate the application of the metal magnetic memory (MMM) technique for investigations on fatigue crack propagation in a ferromagnetic material. Fatigue failure caused by stress concentration is serious in practical engineering. However, early fatigue damages cannot be detected by using traditional nondestructive testing (NDT) methods. Therefore this paper study about NDT method called metal magnetic memory (MMM) that has potentials for evaluating the fatigue damage at the early damage and critical fracture stages. While its capacity to evaluate the distribution of self-magnetic leakage field signals on the component's surface is well-established, there remains a need to scrutinize the physical mechanism and quantitative analysis aspects of this method. To begin with, a fatigue test involving a loading of $7 \mathrm{kN}$ was conducted on a SAE 1045 carbon steel specimen. This material is frequently used in the manufacturing of automotive transmission components that include the axle and spline shaft. MMM signals were measured along a scanning distance of $100 \mathrm{~mm}$ and analysed during the propagation stage. Other than revealing that the value of the magnetic flux gradient signals $d H(y) / d x$ increased in tandem with the crack length, the results also led to the detection of the crack growth location. It was anticipated that the $d H(y) / d x$ value will also exhibit an upward trend with a rise in the fatigue growth rate of $d a / d N$. A modified Paris equation was utilized to correlate $d H(y) / d x$ with $\mathrm{da} / \mathrm{dn}$ through the replacement of the stress intensity factor range $\Delta K$. This resulted in the log-log plot of $d a / d N$ versus $d H(y) / d x$ portraying an inclination similar to the $\log -\log$ plot of $d a / d N$ versus $\Delta K$. A linear relationship was established between $\mathrm{dH}(\mathrm{y}) / \mathrm{dx}$ and $\Delta K$ with the $R^{2}$ value as 0.96 . Players in the automotive industry can benefit from the disclosure that $d H(y) / d x$ can effectively replace $\Delta K$ for the monitoring of fatigue crack growth behaviour.
\end{abstract}

\section{Introduction}

\footnotetext{
* Corresponding author: shahridhwan.jpk@gmail.com
} 
The development of fatigue-safe components is among the most crucial factors in the automotive industry domain. This is attributed to the fact that fatigue failure can lead to accidents, which in turn could result in injuries and loss of life. Automotive transmission components are among those often beset with biaxial fatigue, and SAE 1045 carbon steel is among the most scrutinized materials in this area [1]. The axle and spline shaft are two examples of automotive transmission parts made of SAE 1045 carbon steel where fatigue is the primary cause of failure. This is especially so on the stress raisers [2, 3]. A wide range of studies have been conducted on the fatigue behaviour of this material in the context of automotive components. Pals \& Stephens [4] ascertained the fatigue behaviour of SAE 1045 steel subjected to extremely elevated tensile mean stress for un-notched, mildly notched, and sharply notched test specimens under dissimilar heat treatments. Firat [5] executed a simulation that involved the combined bending and torsion fatigue of a SAE 1045 notched shaft where the design was fashioned to resemble the distinctive geometric and manufacturing features of automotive transmission shafts. Ince \& Glinka [6] on the other hand, investigated the fatigue life of a SAE 1045 notched shaft under multi axial loadings.

Failure deterrence is a significant aspect of automotive engineering. Generally, an inspection for flaws in components by way of non-destructive testing (NDT) is the standard procedure for making certain that a component is in good working order [7]. However, conventional NDT procedures such as ultrasonic testing, radiographic testing, magnetic particle testing, penetrant testing, and eddy current testing are only designed to uncover developed defects. As such, these procedures fall short when it comes to initial fatigue damage prevention [8]. This dilemma can be overcome with the employment of the MMM technique. This technique focuses on identifying the stress concentration zones in order to provide an early indication of damage to a ferromagnetic material [9]. It was first introduced in the 50th international welding conference by the Russian researcher Dubov. The principle of MMM is the magneto-mechanical effects. While equipment working, ferromagnetic material is having self-magnetization phenomenon. When the work load is off the magnetic field is kept down. The residual magnetic fields that remain are related to the mechanical stress. The residual magnetic field density $\mathrm{H}$ is characterized by the normal component $H p(y)$ and tangent component $H p(x)$ on the stress-concentration zones [8].

Hypothetically, the materialization of the magnetic area at the location of highest defect concentration together with the influence of an external magnetic field creates a full demagnetizing field of defects. The entire demagnetizing field that is liberated from the surface of the component is in the form of magnetic scattering fields. The regular component of the scattering field $H(y)$ indicates the location of the metal structure's highest level of inhomogeneity [10]. A substantial number of studies have been performed in efforts to link the MMM signals to fatigue crack growth parameters. Xing et al. [11] investigated the association between the crack growth rate $d a / d N$ and the MMM regular component signal $H(y)$. It was observed that the $H(y)$ increased in tandem with the rise of $d a / d N$ while the correlation curve between $H(y)$ and $d a / d N$ exhibited a sigmoidal figure. Dong et al. [12] described the behaviour of the peak to peak value of $H(y)$ in a tensiontension fatigue crack growth test. According to them, the progression of a fatigue crack coincides with an elevation in the magnetic peak to peak value. Huang et al. [9] conducted dynamic three-point bending fatigue tests to delve into the option of harnessing magnetic flux gradient signals $d H(y) / d x$ for the prediction of fatigue crack propagation. They discovered that $d H(y) / d x$ can be brought into play for determining the degree of stress concentration. They also forwarded that $d H(y) / d x$ is exponential with the loading cycles and just about linear to the crack length.

Due to the shortage of physical models and quantitative defect criteria, the MMM technique is currently deemed a qualitative or semi-quantitative process [13]. As such, its 
capacity to establish a linkage between MMM signals and the fatigue growth parameter remains questionable. The intention of this investigation is to assess the possibility of harnessing the magnetic flux gradient signals $d H(y) / d x$ to ascertain the location of a crack, and to recognize the development of fatigue cracks in materials commonly used in the automotive industry. The authors also intend to determine if the $d H(y) / d x$ can be utilized as a substitute for the stress intensity factor range $\Delta K$ in the Paris equation, an equation frequently used to explain the occurrence of fatigue crack growth.

\section{Experimental set up}

The objective of this experiment is to link the magnetic signal parameter of ferromagnetic materials released during crack propagation to the fatigue crack growth parameters. The fatigue test was conceived in line with the ASTM E647, a typical testing procedure for the measurement of fatigue crack growth rates. This procedure includes the determination of fatigue crack growth rates and the crack driving force of fatigue crack growth, namely, the stress intensity range $\Delta \mathrm{K}$. In the opinion of Dong et al. [12], the successful acquisition of the MMM data signal necessitates the use of a wide specimen to ensure the availability of a satisfactory working space. With this in mind, and in compliance to the ASTM E647 standard, a single edge-cracked specimen with a width of $60 \mathrm{~mm}$, a thickness of $3 \mathrm{~mm}$ and an initial crack length width of $22 \mathrm{~mm}$ was employed. The specimen was made of SAE 1045 carbon steel which is extensively used in automotive transmission parts [2]. The previously executed tensile test on the SAE 1045 carbon steel material disclosed that its ultimate tensile strength is $611.7 \mathrm{Mpa}$, its yield stress is $363.5 \mathrm{Mpa}$, and its elastic modulus is $206.7 \mathrm{GPa}$.

The constant amplitude fatigue test was performed on the SAE 1045 carbon steel specimen using the $100 \mathrm{kN}$ servo-hydraulic machine with a loading of $7.0 \mathrm{kN}$. Our previous work in this area established that this testing circumstance is appropriate for the scrutiny of crack growth propagation as well as for realizing correlation between the magnetic flux leakage signals and the fatigue crack growth parameter. The crack opening displacement (COD) device was employed to measure the propagation of crack length on the specimen, and the MMM scanning device TSCM-2FM was utilized to capture the magnetic flux leakage signals. Upon the arrival of the crack in the specimen at the pre-set length, the fatigue test was halted for the measurement of the magnetic flux leakage signal. The sensor of the MMM device rolled on the determined scanning line with the gap between the scanning line and the initial crack set at $20 \mathrm{~mm}$. All related data were documented up to the point where the specimen failed. Figure 1 exhibits the schematic geometry of the specimen in relation to the scanning line, the MMM device, and the installation of the fatigue test involving the COD device.

\section{Equations and mathematics}

The concepts of linear elastic fracture mechanics were applied to examine the fatigue crack growth process stretching from initial crack detection to ultimate failure. The fundamental supposition here is that during the progression of fatigue, the state of the material is, for the most part, linear elastic. This examination entails an establishment of the linkage between the crack growth rate $d a / d N$ and the stress intensity factor range $\Delta K$. Generally, the stress intensity factor range is used for describing the behaviour of fatigue crack growth in metals and alloys under constant amplitude test conditions [14]. Fundamentally, the $d a / d N$ can be ascertained through the slope of the graph for crack length a against the number of cycles $\mathrm{N}$. The standard log-log plot of $d a / d N$ versus $\Delta K$ reveals that the fatigue crack growth 
process can be separated into three areas. Among them, the second area frequently displays a linear linkage between $\log d a / d N$ and $\log \Delta K$. This can be expressed through the Paris law equation as in Equation (1).

$$
d a / d N=C(\Delta K)^{m}
$$

where coefficient $C$ and exponent $m$ are the material constants [14]. During this endeavour, we made an adjustment to Equation 1 to realize an innovative relationship between $d H(y) / d x$ and $d a / d N$.

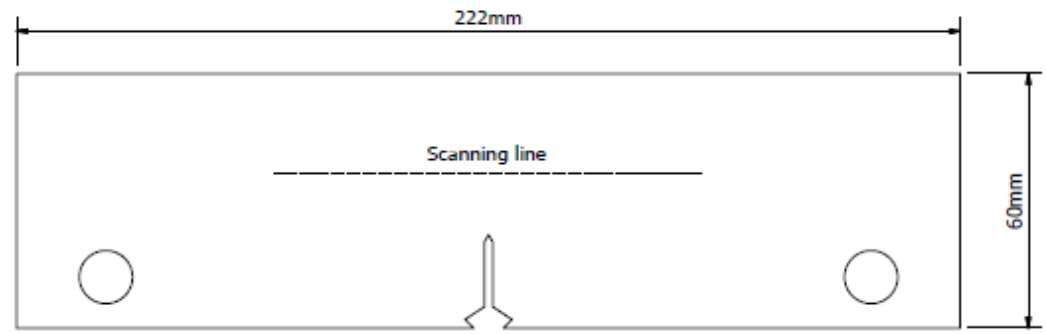

(a)

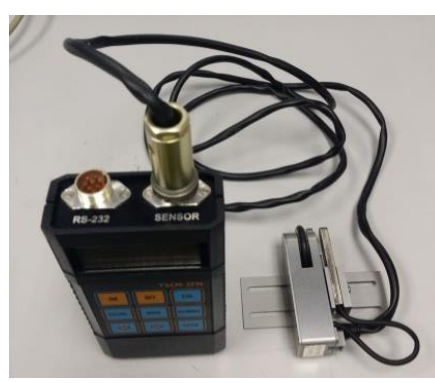

(b)

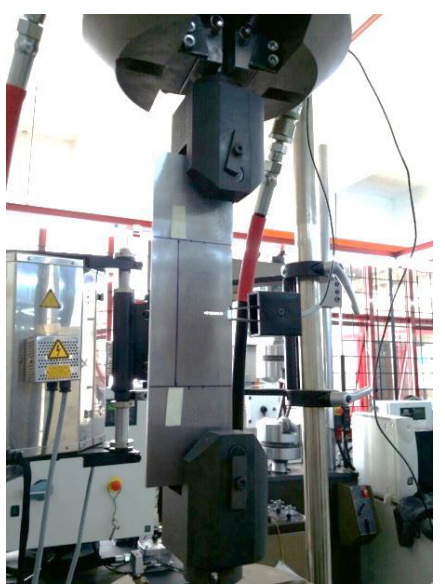

(c)

Fig. 1. Experimental setup (a) schematic geometry of specimen with scanning line; (b) MMM device; (c) installation of fatigue test with COD device.

\section{Results and discussion}

\subsection{Fatigue Crack Growth Behaviour of SAE 1045 carbon steel}

The fatigue test revealed that the $\log -\log$ plots of $d a / d N$ versus $\Delta K$ assume a sigmoidal shape that can be separated into three regions. This situation is displayed in Figure 2. At Region I, the $d a / d N$ and $\Delta K$ values are extremely low and the early stage of the crack is apparent at the initial crack length. Region II is deemed the intermediate region of the entire fatigue crack growth process. Here, the propagation of the crack is stable. In line with the Paris equation, the relationship representing the straight line is $d a / d N=3 \mathrm{E}-9(\Delta K)^{3.0967}$. 
According to this equation, the value of coefficient $C$ is 3E-9 while the value of slope $m$ is 3.0967. The $R^{2}$ value of 0.9796 indicates that the mathematical equation offers a plausible description of the association between $\log d a / d N$ and $\log \Delta K$. As can be observed in Figure 2, crack propagation occurs within Region II from $2 \mathrm{~mm}$ to $18 \mathrm{~mm}$. In Region III, the $d a / d N$ values rise briskly and the value of $\Delta K$ is at an exceptionally elevated level. Crack growth is unstable in this region and crack fracture in the specimen ultimately occurs at the crack length of $20 \mathrm{~mm}$.

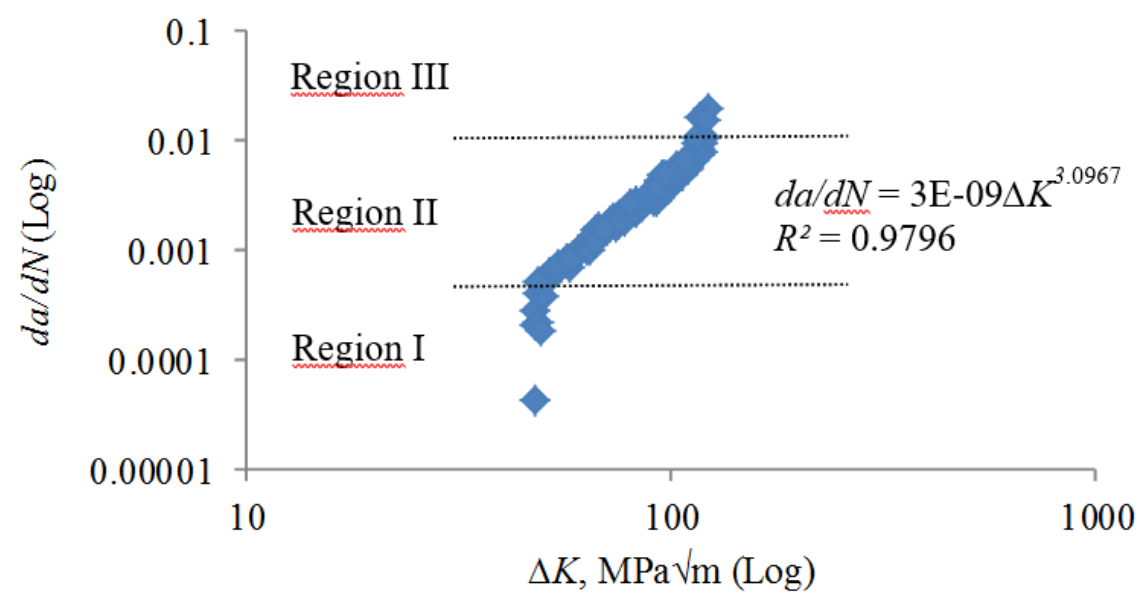

Fig. 2. The relationship between crack growth rate, $d a / d N$ and stress intensity factor range, $\Delta K$

\subsection{Observation of magnetic flux gradient signals, $d H(y) / d x$}

The magnetic flux gradient signals $d H(y) / d x$ were captured during the fatigue test. Figures 3(a) to 3(c) exhibit the magnetic flux gradient signals $d H(y) / d x$ during the propagation stage in which the crack length stretches from $2 \mathrm{~mm}$ to $18 \mathrm{~mm}$. The figures portray alterations in the $d H(y) / d x$ value as the MMM device sensor rolled along the scanning distance of 100 $\mathrm{mm}$.

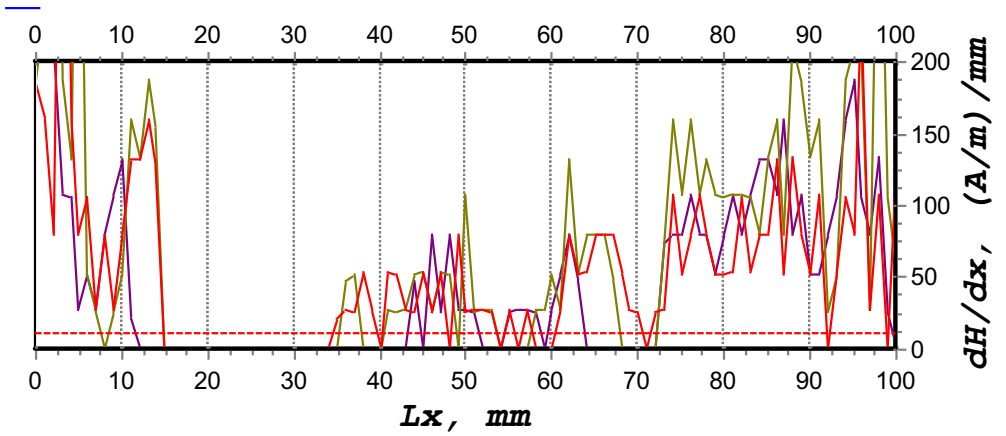

(a) 


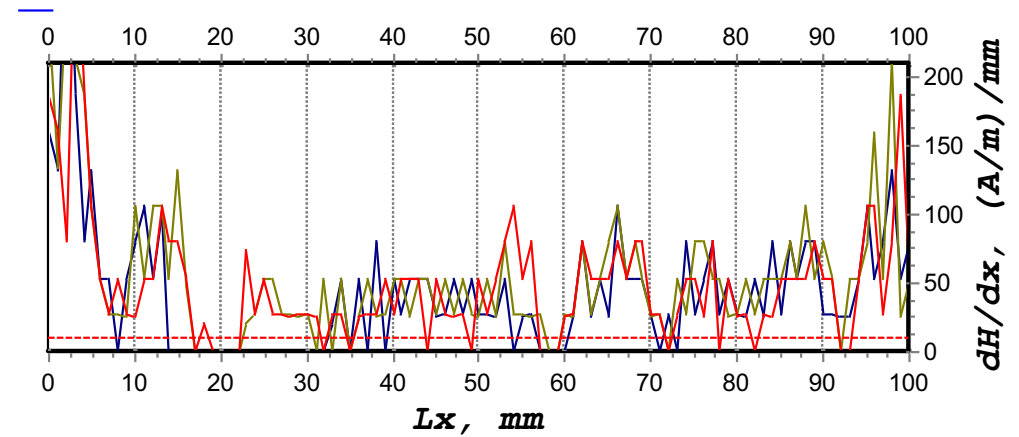

(b)

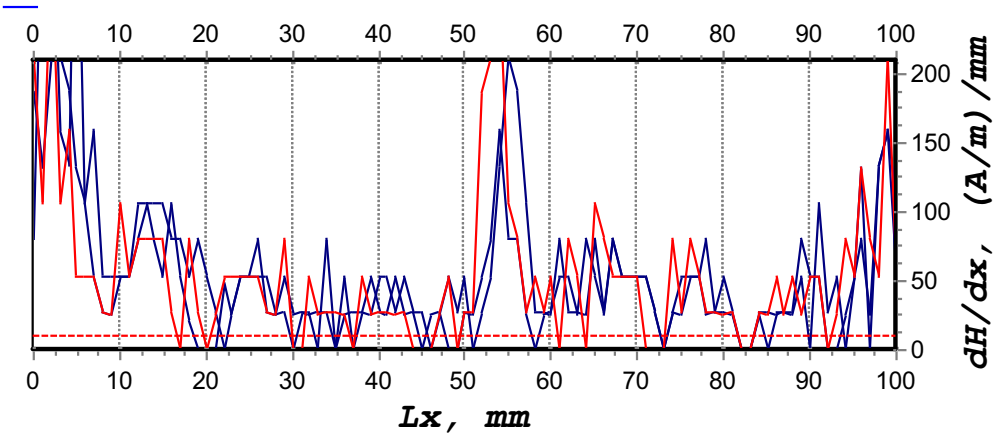

(c)

Fig. 3. The $d H(y) / d x$ signals response at different length of crack propagation (a) $2-6 \mathrm{~mm}$; (b) $8-12$ $\mathrm{mm}$; (c) $14-18 \mathrm{~mm}$.

As illustrated in Figures 3(a) to 3(c), at the scanning distance of $50 \mathrm{~mm}$ to $60 \mathrm{~mm}$, the value of $d H(y) / d x$ rose in tandem with increases in the crack length. It is assumed that this represents the crack growth location. A previously conducted study disclosed that the $d H(y) / d x$ can be utilized for describing the degree of stress concentration, as well as for determining the location of the crack $[15,16]$. The notch initiates the occurrence of stress concentration which sets off the propagation of the fatigue crack. Eventually, the progression of the crack culminates in definitive material failure [4].

As portrayed in Figures 3(a) to 3(c), at a scanning distance of 0 to $50 \mathrm{~mm}$, and 60 to 100 $\mathrm{mm}$, the value of $d H(y) / d x$ is fairly high. We presume that this is attributed to the influences of the original magnetic field from the fatigue testing machine clamps, as well as the environmental magnetic field $[17,18]$. The $d H(y) / d x$ emanating from the crack growth of the specimen become increasingly apparent with extensions in the length of the crack. This is illustrated in Figure 3(c).

Investigations were also conducted on regular alterations occurring at the scanning distance of $50 \mathrm{~mm}$ to $60 \mathrm{~mm}$. Table 1 displays the $d H(y) / d x$ values attained for different crack lengths at a scanning distance of $50 \mathrm{~mm}$ to $60 \mathrm{~mm}$.

Table 1. $d H(y) / d x$ value in crack propagation region at a scanning distance of 50-60 $\mathrm{mm}$.

\begin{tabular}{|c|c|c|c|c|c|c|c|c|c|}
\hline $\begin{array}{c}\text { Crack length } \\
(\mathrm{mm})\end{array}$ & 2 & 4 & 6 & 8 & 10 & 12 & 14 & 16 & 18 \\
\hline $\begin{array}{c}d H(y) / d x \text { value } \\
((\mathrm{A} / \mathrm{m}) / \mathrm{mm})\end{array}$ & 27 & 27 & 27 & 54 & 80 & 107 & 160 & 213 & 267 \\
\hline
\end{tabular}


The table reveals that the $d H(y) / d x$ value rose in tandem with increases in the length of the crack. However, at the crack length of $2 \mathrm{~mm}$ to $4 \mathrm{~mm}$, the $d H(y) / d x$ values remained unaltered. This could be attributed to the distance of the set scanning line being slightly too remote from the location where the crack occurred. In such a circumstance, the sensor would fail to detect alterations in the values of the $d H(y) / d x$. Upon the arrival of the crack line at $8 \mathrm{~mm}$, the crack location was drawn closer to the scanning line, and from then on, increases in the $d H(y) / d x$ values were consistent. At the crack length of $18 \mathrm{~mm}$ the $d H(y) / d x$ value is exceptionally elevated as the specimen is on the brink of experiencing an ultimate fracture. The experimental results disclosed that the MMM sensor device was unable to provide satisfactory $d H(y) / d x$ values at the distance of $2 \mathrm{~mm}$ to $4 \mathrm{~mm}$. In view of this, all following investigations were confined to the utilization of $d H(y) / d x$ values at the distance of between $6 \mathrm{~mm}$ and $18 \mathrm{~mm}$.

It is believed that $d H(y) / d x$ related to the crack length and based on the previous study, Huang et al [9] and Chongchong et al [13] established that a linear correlation existed between the crack length and $d H(y) / d x$. Figure 4 portrays the linear relationship between $d H(y) / d x$ and the crack length at the propagation stage of this study. With the $R^{2}$ value at 0.971 , the relationship between the $d H(y) / d x$ signals and the crack length is deemed adequate.

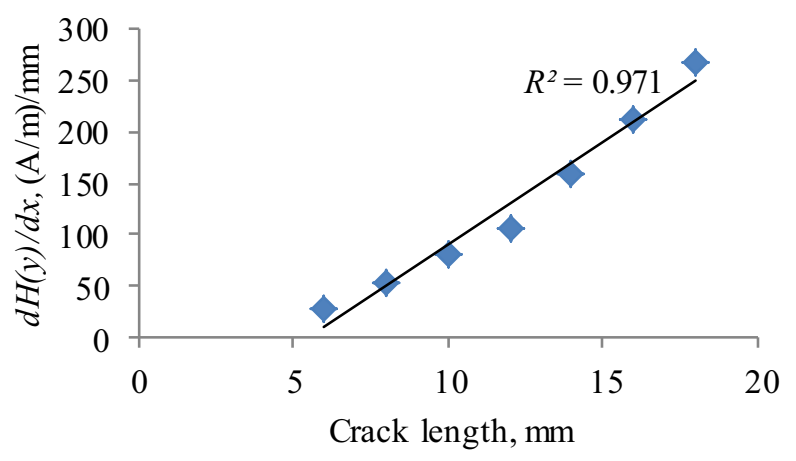

Fig. 4. The relationship between the magnetic flux gradient signals, $d H(y) / d x$ and crack length.

\subsection{Relationship between magnetic flux gradient signals, $d H / d x$ and stress intensity factor range, $\Delta K$}

The MMM technique is a non-destructive procedure for ascertaining stress concentration zones. These zones are the major locations for the onset of failure growth [10]. The $d H(y) / d x$ captured by means of the MMM technique can be used to express the fatigue crack growth by replacing $\Delta K$ in the Paris equation with $d H(y) / d x$. Equation 2 below represents the proposed modified Paris equation:

$$
d a / d N=C(d H(y) / d x)^{m}
$$

The log-log plot of $d a / d N$ against the $d H(y) / d x$ signals during the propagation stage is exhibited in Figure 5. As displayed, the pattern of this plot is similar to that of the second region in Figure 2. The $d H(y) / d x$ value increased in tandem with the rise in $d a / d N$. This indicates that the variation of $d H(y) / d x$ signals reflects the alteration of $\Delta K$ during the crack growth process. The linear relationship between $d a / d N$ and $d H(y) / d x$ is expressed as $d a / d N=5 \mathrm{E}-5(d H(y) / d x)^{0.8893}$. According to the equation, the value of coefficient $C$ is $5 \mathrm{E}-5$ while the value of the slope $m$ is 0.8893 . The $R^{2}$ value attained from the relationship is 
0.9554. This signifies that the correlation between $d a / d N$ and $d H(y) / d x$ is acceptable. Hence, the fatigue crack growth rate can be assessed and foreseen through the correlation between $d a / d N$ and $d H(y) / d x$.

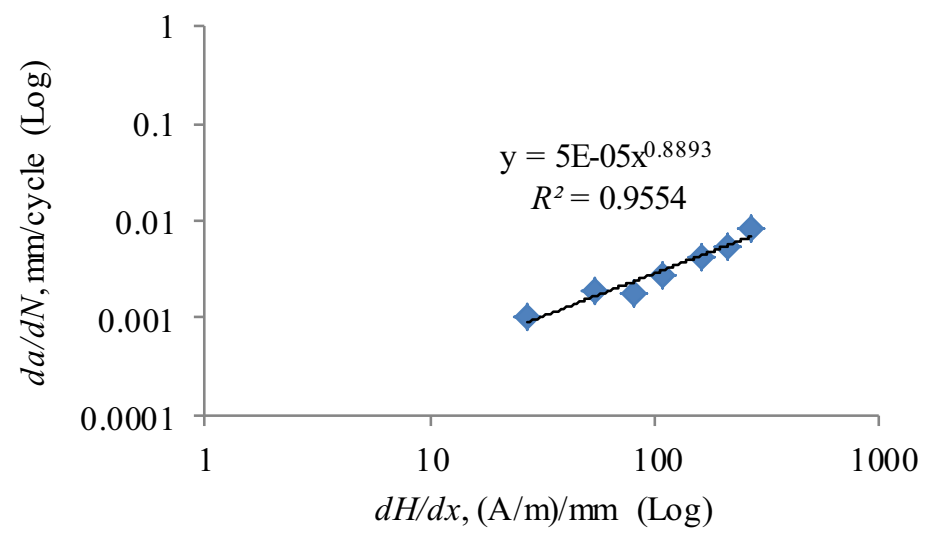

Fig. 5. The relationship between the crack growth rate, $d a / d N$ and magnetic flux gradient signals, $d H(y) / d x$

Hypothetically, $d H(y) / d x$ and $\Delta K$ are similar in the sense that they both indicate the state of stress during fatigue crack growth. Xing et al. [11] and Ahmad et al. [19] investigate relationship between $\Delta K$ and $H(y)$ and it was stated that $H(y)$ variation reflects the change of stress intensity factor $\Delta K$ during the whole crack growth process and linear relation was represented between $\Delta K$ and $H(y)$ graph. Figure 6 illustrates the investigation conducted on the relationship between $d H(y) / d x$ and $\Delta K$. The log-log plot of $d H(y) / d x$ versus $\Delta K$ reveals that the relationship between $d H(y) / d x$ and $\Delta K$ is linear. The acquired $R^{2}$ value of 0.9612 verifies that the relationship between the $d H(y) / d x$ signals and $\Delta K$ is acceptable. As such, $d H(y) / d x$ has the potential to replace $\Delta K$ during efforts to evaluate the fatigue crack growth process.

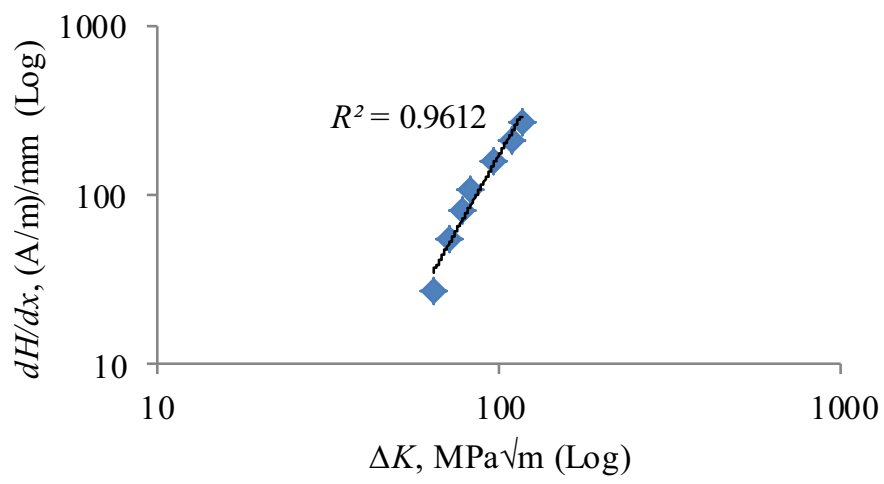

Fig. 6. The relationship between the crack growth rate, $d a / d N$ and magnetic flux gradient signals, $d H(y) / d x$

\section{Conclusion}

This investigation probes the likelihood of employing the MMM technique for the evaluation of fatigue crack growth in a ferromagnetic material. The fatigue crack growth 
behaviour of SAE 1045 carbon steel is expressed by way of the log-log plot of $d a / d N$ versus $\Delta K$. The linear relationship obtained in Region II is represented by the Paris equation where the value of coefficient $C$ is $3 \mathrm{E}-9$ and the value of the slope $m$ is 3.0967. The magnetic flux gradient signals $d H(y) / d x$ captured by way of the MMM device sensor disclosed that at the scanning distance of $50 \mathrm{~mm}$ to $60 \mathrm{~mm}$, the $d H(y) / d x$ value increased along with extensions in the crack length. The $d H(y) / d x$ value analysed at that range confirmed that the relationship between $d H(y) / d x$ and the crack length is linear. The results from this investigation also suggest that it is possible to detect the location of a crack through the utilization of $d H(y) / d x$. A modified Paris equation entailing the replacement of the stress intensity factor range $\Delta K$ with $d H(y) / d x$ was recommended by this study. The loglog plot of $d a / d N$ versus $d H(y) / d x$ displays a similar curve trend to that of $d a / d N$ versus $\Delta K$. According to the equation, the value of coefficient $C$ is $5 \mathrm{E}-5$ while the value of slope $m$ is 0.8893. The outcomes from this investigation confirm that $d H(y) / d x$ can be utilized for describing the fatigue crack behaviour of SAE 1045 carbon steel, a commonly used material in the manufacturing of automotive transmission components. Nonetheless, further investigations ought to be in the pipeline regarding the accuracy of the magnetic signals, the capacity of these signals to provide an early indication of crack development, and the applicability of the MMM technique in the automotive industry.

The authors would like to express their gratitude to Universiti Kebangsaan Malaysia and Ministry of Education Malaysia for funding under the grant of FRGS/2/2014/TK01/UKM/02/03 for supporting this project research.

\section{References}

1. Y. Verreman and H. Guo, Fatigue \& Fracture of Engineering Materials \& Structures, 30, 932-946 (2007)

2. M. Y. Noordin, V. C. Venkatesh, S. Sharif, S. Elting, and A. Abdullah, Journal of Materials Processing Technology, 145, 46-58 (2004)

3. A. Guimaraes, P. Brasileiro, G. Giovanni, L. Costa, and L. Araujo, Engineering Failure Analysis (2016)

4. T. Pals and R. Stephens, International journal of fatigue, 26, 651-661 (2004)

5. M. Firat, Finite Elements in Analysis and Design, vol. 54, 16-27 (2012)

6. A. Ince and G. Glinka, International Journal of Fatigue, 62, 34-41(2014)

7. V. L. de Araújo Freitas, V. H. C. de Albuquerque, E. de Macedo Silva, A. A. Silva, and J. M. R. Tavares, Materials Science and Engineering, 527, 4431-4437(2010)

8. H. Y. Xing, D. B. Wu, and M. Q. Xu, Advanced Materials Research, 408-411 (2011)

9. H. Huang, S. Jiang, R. Liu, and Z. Liu, Journal of Nondestructive Evaluation, 33, 407$412(2014)$

10. A. Dubov, Metal Science and Heat Treatment, 39, 401-405 (1997)

11. H. Y. Xing, R. X. Wang, M. Q. Xu, and J. Z. Zhang, Key Engineering Materials, 2293-2296 (2007)

12. L. H. Dong, B. S. Xu, H. P. Wang, and N. Xue, Applied Mechanics and Materials, 190, 415-418 (2012)

13. L. Chongchong, D. Lihong, W. Haidou, L. Guolu, and X. Binshi, Journal of Magnetism and Magnetic Materials, 405, 150-157 (2016)

14. C. A. R. P. Baptista, A. Adib, M. Torres, and V. Pastoukhov, Mechanics of Materials, 51, 66-73 (2012)

15. S. Changliang, D. Shiyun, X. Binshi, and H. Peng, NDT \& E International, 43, 8-12, (2010) 
16. Y. Zhang, R. Gou, J. Li, and G. Shen, Frontiers of Mechanical Engineering in China, 5, 226-232 (2010)

17. J. Leng, M. Xu, S. Zhao, and J. Zhang, Measuring Technology and Mechatronics Automation, 784-786 (2009)

18. H. Xing, Y. Dang, B. Wang, and L. Jiancheng, Chinese Journal of Mechanical Engineering, 1-6 (2016)

19. M. Ahmad, A. Arifin, and S. Abdullah, Journal of Mechanical Engineering and Sciences, 9, 1727-1733 (2015) 